\title{
High Throughput Fabrication Process of a Zernike Phase Plate
}

\author{
Y. Konyuba $^{1}$, H. Iijima ${ }^{1}$, Y. Abe ${ }^{2}$, M. Suga ${ }^{1}$, and Y. Ohkura ${ }^{1}$ \\ 1. JEOL Ltd., 3-1-2 Musashino, Akishima, Tokyo 196-8558, Japan \\ 2. Yamagata Research Institute of Technology, Matsuei 2-2-1, Yamagata, 990-2473, Japan
}

In transmission electron microscopy (TEM) for biological and polymer samples, it is difficult to image with high contrast, since they are mostly composed of light elements and have similar density. One solution to enhance the contrast is utilization of phase contrast microscopy, which is realized in optical microscopy. Accordingly, many types of phase plates for electron microscopy have been proposed. Zernike phase contrast TEM (ZPC-TEM) with a Zernike phase plate (ZPP) provides higher contrast at Af (defocus) $=0$ with respect to one in conventional TEM [1]. ZPC-TEM attracts much attention in Cryo-TEM applications such as cryo-electron tomography and single-particle analysis [2], because their specimens are easy to be damaged with electrons and they need high contrast with minimum dose on the specimen.

So far, the ZPP is carefully made by hands. Therefore, production throughput is low. Recently, a ZPP fabricated on silicon wafers has been reported [3-4]. However, the entire throughputs of their processes were low, since a center hole was formed with focused ion beam (FIB), which made the processes intermittent. Moreover, resulting shapes of the center hole by these processes showed imprecise circles.

To solve these problems, we have tried to fabricate the ZPPs with a process, which have been used in the field of micro electro mechanical systems (MEMS) technology. As a first trial, we tried to make titanium (Ti) / silicon nitride ( $\mathrm{SiN}$ ) / Ti sandwich type ZPPs. A process adopted is shown in Fig. 1. In this process, a center hole was fabricated in the SiN films by electron beam lithography and dry etching. Finally, to give electron conductivity to the plate, the top and bottom surfaces of the phase plate were coated with Ti. By virtue of this continuous process, the center hole fabricated was nearly perfect circular as shown in Fig. 2. This process opens the way to make the ZPPs with accurate structure with high throughput.

We compared an image of carbon thin film (Quantifoil) taken with the Ti/SiN/Ti ZPP with the one without any phase plate as shown in Fig 3(a) and 3(b). These images were observed with a field emission TEM (JEM-2200FS) at $200 \mathrm{kV}$. From these images, we confirmed that the increase in low spatial frequency contrast in the Fourier transform of the TEM image using the Ti/SiN/Ti ZPP and that the increase in contrast in the TEM image using the phase plate. At this stage, the ZPP shows the decrease in high frequency as you see in Fig 3.

To improve the stability and optical characteristics of the phase plate, we are currently trying to fabricate the ZPPs with thinner Ti/SiN/Ti films. We have also started the fabrication of the ZPPs with amorphous carbon films with the similar process.

\section{References}

[1] Danev, R., et.al, Ultramicroscopy, 88, (2001), 243-252.

[2] Nagayama, K., Journal of electron microscopy 60, (2011), 43-62.

[3] Marko, M., et al, Journal of structural biology, 184, (2013), 237-244. 
[4] Kuo, P. C., et al, ACS nano, 7, (2013), 465-470.

Silicon nitride deposition

on both sides

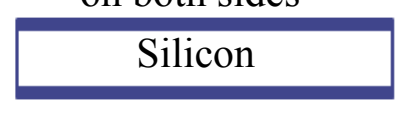

Lithography of

the window pattern
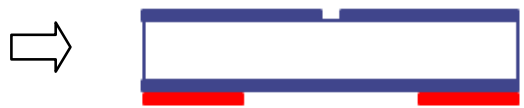

Titanium deposition on top side
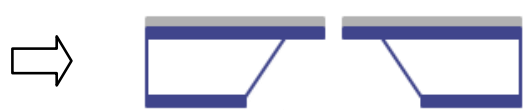

Lithography of the center hole pattern

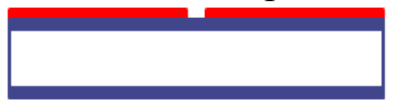

Silicon nitride patterning by dry etching
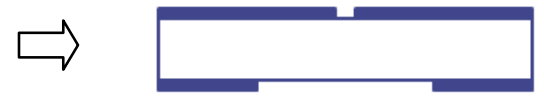

Titanium deposition on bottom side

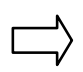

Silicon nitride patterning by dry etching
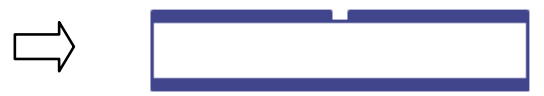

Silicon removal by wet etching

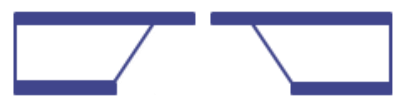

Fig.1 The fabrication process of a Ti/SiN/Ti Zernike Phase Plate using micro electro mechanical systems (MEMS) technology. The center hole was also fabricated using lithography and dry etching. FIB was not used for the fabrication of the hole.
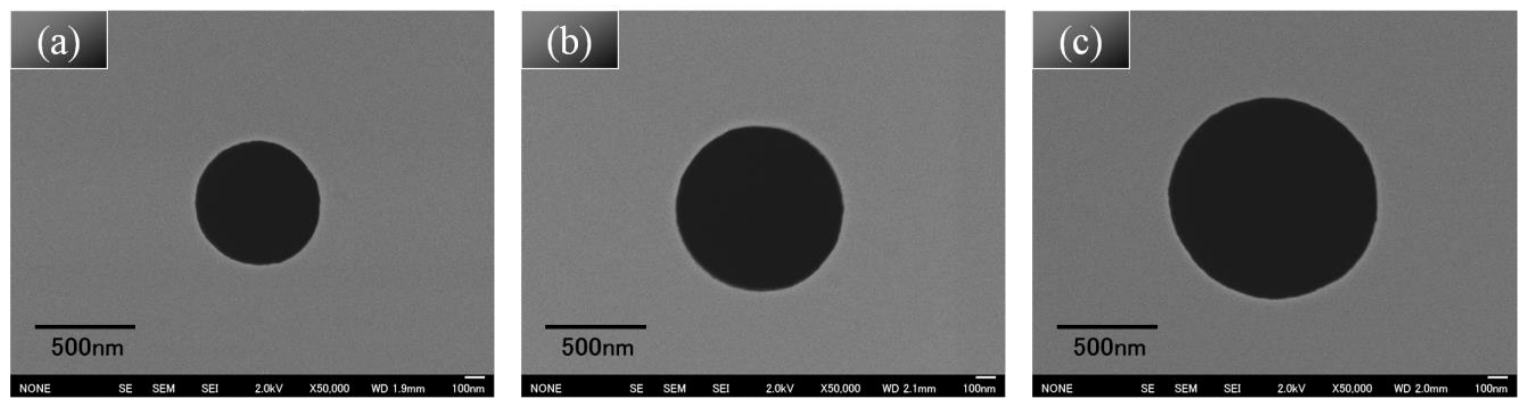

Fig.2 SEM images (secondary electron) of the center holes in the Ti/SiN/Ti Zernike Phase Plates. Hole diameters are approximately $0.6 \mu \mathrm{rn}$ (a) $0.8 \mu \mathrm{rn}$ (b) and $1.0 \mu \mathrm{rn}$ (c).
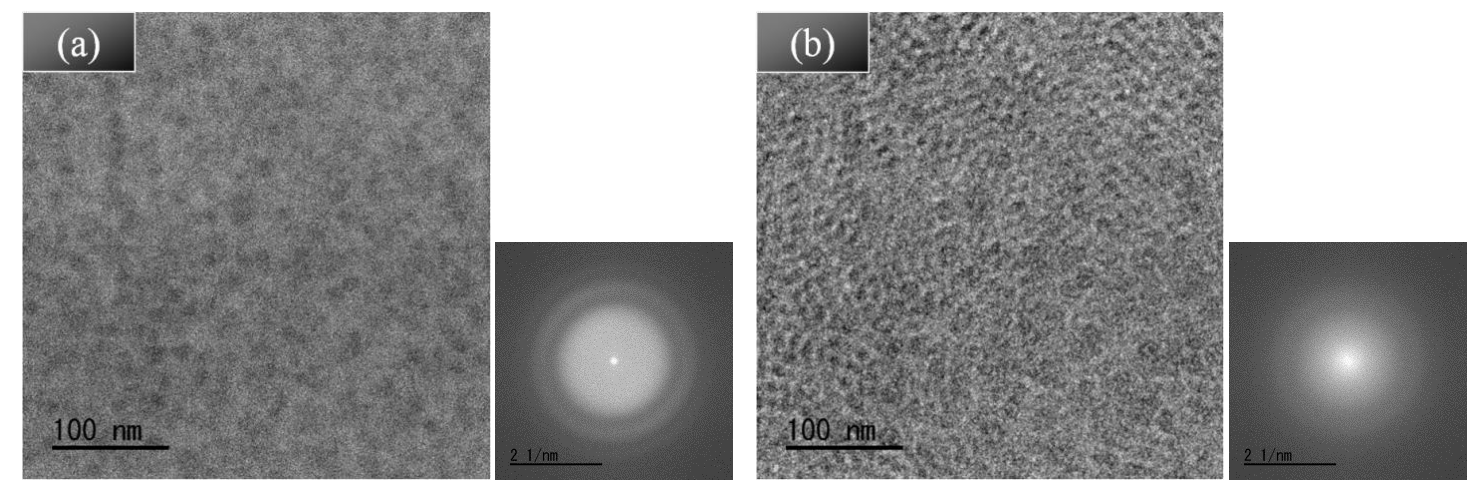

Fig.3 Comparison of a conventional TEM image (a) with the Ti/SiN/Ti Zernike phase contrast TEM image (b). The Fourier transforms of these images are also shown. 